\title{
THE ATTENTIONAL COSTS OF INTERRUPTING TASK PERFORMANCE AT VARIOUS STAGES
}

\author{
Christopher A. Monk \& Deborah A. Boehm-Davis \\ George Mason University \\ Fairfax, VA
}

\author{
J. Gregory Trafton \\ Naval Research Laboratory \\ Washington, DC
}

\begin{abstract}
The visual occlusion technique has received considerable attention in recent years as a method for measuring the interruptible aspects of in-vehicle information system (IVIS) task performance. Because the visual occlusion technique lacks a loading task during "occluded" periods, an alternate method was adopted to provide increased sensitivity to the attentional costs of interruptions on IVIS-style task performance. Participants alternated between performing a VCR programming task and a simple tracking task. Results indicate that it does matter at which point the VCR task is interrupted in terms of time to resume the VCR task. Specifically, the resumption time, or lag, was lowest right before beginning a new task stage such as entering the show endtime, or when performing a repetitive scrolling task. The results suggest that it might be appropriate to include measures of resumption lag when testing the interruptability of IVIS-style tasks.
\end{abstract}

One of the key challenges for in-vehicle information system (IVIS) designers is to build user interfaces that are conducive to multi-tasking, thereby minimizing driver distraction. One way to think about this problem is in terms of task interruption. IVIS task performance is continually interrupted by the primary task of driving (as well it should be). Drivers must resume their IVIS task after attending to the driving task for some time, and this continues back and forth until the IVIS task is complete. Typically, the exposure periods to the IVIS task are in the realm of two seconds or less (Wierwille, 1993; Gellatly \& Kleiss, 2000). As a result, the ability drivers' ability to perform task elements during these short exposures is critical to the design of safe IVIS devices. An interesting consideration is when a given IVIS task is most interruptible. In other words, at what stages during the IVIS task will the driver best be able to recover from an interruption in terms of time and performance. The purpose of this study was to identify any differential attention switching costs for interruptions occurring at various task stages.

To answer this question, there is a need for a task measure that will allow interface designers to identify interruptible tasks and more importantly to distinguish between interruptible and uninterruptible tasks. One method that has garnered a great deal of attention in recent years for addressing this problem is the visual occlusion technique, which has been proposed as a method for detecting the interruptability of IVIS tasks (Green \& Tsimhoni, 2001; Karlsson \& Fichtenberg, 2001).
The visual occlusion technique creates an environment where a driver's vision is alternatively available or blocked while completing an IVIS task. This switching on-and-off of vision is an attempt to emulate the visual attention distribution of a driver operating an IVIS device while driving. In other words, the visual occlusion technique is designed to simulate a driver's alternatively switching visual attention to the road and to the device while multi-tasking. However, a problem with the visual occlusion technique in this application is its lack of a task load while vision is occluded. This is not representative of real-world conditions where drivers perform several tasks while looking at the road, such as monitoring the road and traffic and looking for navigational cues.

Because the visual occlusion technique does not incorporate a task during the occluded periods, it is difficult to know if subjects are able to resume the IVIS task without any attention switching latency. Subjects are free to maintain their task goal state during the occluded periods without interference from another driving related task, unlike in the real driving situation. As a result, the technique generates a prediction of performance in a dual-task setting that fails to account for any attentional cost when switching back and forth between two tasks. Because of this shortcoming, an alternative task paradigm was employed to study where IVIS-style tasks are best interrupted. This method functioned similarly to the visual occlusion technique, but it presented subjects with a loading task during the occluded periods. A VCR programming task was used as the IVIS-style task because it maps well to in-vehicle 
destination entry type tasks. Programming a show in the VCR includes entering program elements like the show start-time and end-time. These program elements are similar to the destination entry elements of street name, street number, and city.

In addition to this revised methodology, an appropriate measure of interruptability was required. One of the key measures of the visual occlusion technique is the Total Shutter Open Time (TSOT), which is the sum of the intervals that the driver's vision is "available" (see Figure 1). In the interruptions research paradigm, there is a measure of task resumption time that is referred to as the resumption lag (Altmann \& Trafton, 2002). This lag is the additional time it takes users to resume the task after having switched back from an interruption. As shown in Figure 2, the resumption lags take away from the time-on-task during an open period in the occlusion cycle, thereby potentially increasing the overall TSOT measure. In other words, longer resumption lags result in less time-on-task, which in turn results in the need for more shutter open time. As such, the resumption lag measure may provide designers with key information about how to reduce TSOT, and thereby reduce eyes-off-road time.

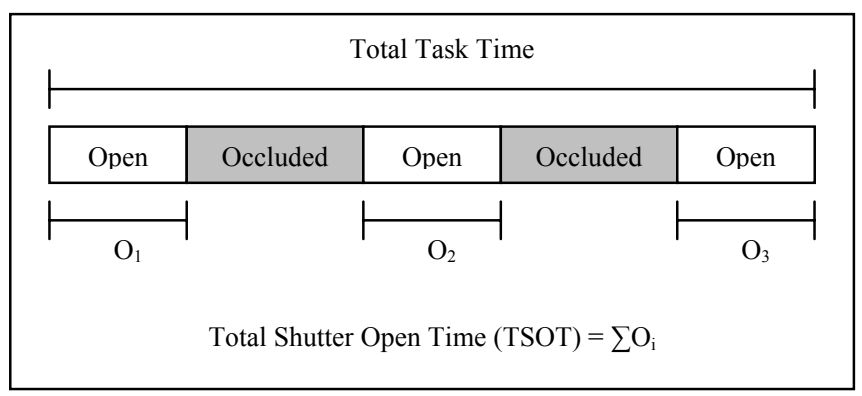

Figure 1. Visual Occlusion Technique Timeline

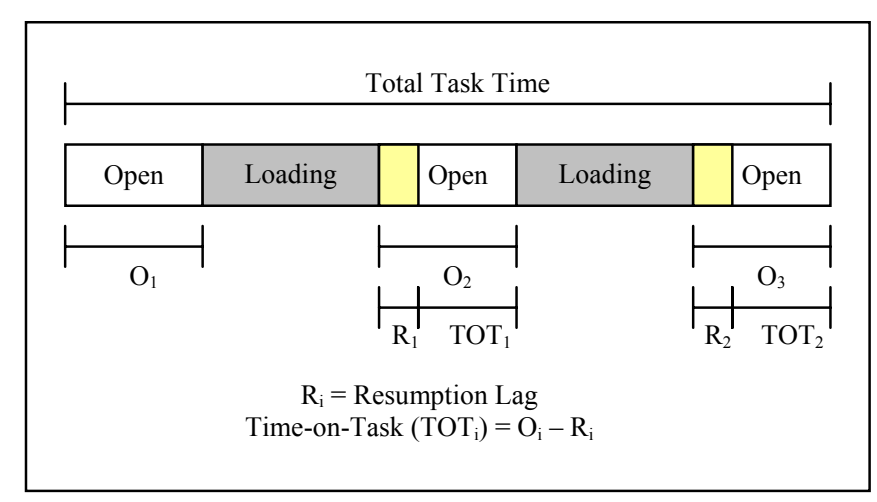

Figure 2. Revised Occlusion Timeline with Effects of Loading Task

By measuring the resumption lag as the attention switching cost when performing two tasks in an interlaced fashion, more accurate explanation of total task times will be possible. In addition, appropriate characterization of the interruptible of IVIS task stages will be quantifiable in terms of resumption lags.

The benefit of using the simulated VCR was that it consisted of four basic tasks that each subdivides nicely into four task stages, or interruption points. These four tasks were entering the start-time, end-time, day of week, and channel number. The first interruption point was just before beginning a new task such as entering the program start time. The next interruption point was anytime while the user was in the middle of performing the task, or entering the start-time in this example. The third interruption point was just before concluding the task, such as saving the show start-time to the program. Finally, there was one task stage that was treated separately from the mid-task performance stage because it involved repetitive inputs such as scrolling through a list. This interruption point was hypothesized to be highly interruptible due to its repetitive nature.

The switching between the VCR and loading task was time-based rather than task-based, so the series of interruption points was never exactly the same for each trial. However, the interruptions were frequent enough to provide an adequate sample of resumption lags for each of the four interruption points across all trials. Because this task paradigm did not allow for strict control over when the VCR task was interrupted, the resumption lags were classified into the four interruption points from the task log files after the fact. It is important to note that although the series of interruption points was never the same from trial to trial, the coding of the task stages where the interruptions occurred was the same. For example, an interruption occurring just before beginning the end-time entry task was always coded as a start-task resumption lag.

\section{METHOD}

\section{Participants}

Twenty-one undergraduates from the George Mason University psychology subject pool participated in this study as part of a course requirement. Six men and 15 women participated and ranged in age from 17 to 22 , with an average age of 19 years. The 17 -year-old participant provided parental consent prior to participating.

\section{Tasks}

The IVIS-style task was a simulated VCR programming task on a Macintosh G4 computer with a 17-inch VGA monitor. Programming a show in the VCR included using the mouse to enter the show start-time, 
end-time, day of week, and channel number. All interactions with the VCR were based on simulated VCR buttons; there were no field entries. To enter a starttime, the participant was required to click on the starthour button, then repeatedly click on the up or down arrow until the displayed hour number reached the target. Next, the participant clicked on the 'Enter' button to save the start-hour setting. The participant was then required to repeat the same steps for the start-10 minute and start-minute settings. The same process was completed for the end-time, day of week, and channel number entries, respectively. The VCR display was blank when no setting was selected. The participants were not required to memorize the show information to be programmed. Instead, the show name, start-time, end-time, day of week, and channel number were posted to the right of the monitor on a $3 \times 5$ index card.

The tracking task required the participant to keep the mouse cursor over an airplane moving around the screen in a random pattern. The view of the airplane was as if from behind, so the movement was horizontal and vertical. The easy and hard settings for the tracking task were defined solely by the speed at which the airplane moved in its random pattern. The airplane moved much faster in the hard condition than in the easy condition. Neither condition was overwhelmingly difficult for the participants; although they were difficult enough that close attention was required to perform the task successfully. The VCR task was on the left side of the monitor and the tracking task was on the right side. Only one side was visible to the subject at any given time.

\section{Design}

The primary task was the VCR task where participants programmed show information for five seconds at a time. After five seconds, the VCR task was then interrupted by the tracking task for 3,8 , or 13 seconds, alternating back and forth until the VCR program show was completed. The tracking task that was either slow (easy) or fast (hard) within each program show trial. This cycle of five seconds on the VCR task interlaced with the tracking task continued until the VCR program entry was completed. The result was a $4 \times 2$ within-subjects design. The two factors were the four VCR task stages, or interruption points (starttask, mid-task, end-task, and scroll), and tracking task difficulty (easy and hard). The tracking difficulty factor was included to determine if the nature of the loading task had any effect on the resumption lag or other task performance measures. The primary dependent measure was resumption lag, which was the response time after switching from the tracking task to the first mouse-click on a VCR button. The total VCR task time, not including interruption times, was also measured. Each participant completed one trial in each of the six experimental cells. The show and trial orders were randomized for each participant.

\section{Procedure}

Each participant was instructed on the VCR task first and then given two practice trials with the VCR task only. Participants programmed the same show information for each practice trial because the intention was to familiarize the participants with the task. After completing the practice with the VCR task, the participants were given two 60 -second practice trials with the tracking task alone. The participants were then introduced to the switching trials, where they spent five seconds on the VCR task and then five seconds on the tracking task, alternating back and forth until the VCR program show was completed. A new show was introduced for the two switching practice trials. After the two practice switching trials, the participants completed six experimental trials, each with new show information to be programmed. Finally, the participants were debriefed and dismissed.

\section{RESULTS}

As predicted, resumption lags were consistently present when the VCR task was paired with the tracking task in an alternating fashion. The resumption lags averaged just over 1600 milliseconds (ms) across all conditions. Despite successful pilot testing with the easy and hard tracking settings, the differences did not materialize during the full experiment. There were no stable differences between the easy and hard conditions for the resumption lag measure, so the data were collapsed across this factor for the analysis of the interruption points factor. The likeliest explanation is that neither tracking task setting was demanding enough to prevent participants from rehearsing their task state while performing the tracking task.

All resumption lags were coded according to their corresponding interruption point for each participant. These data were entered into a repeated-measures analysis of variance (ANOVA) with four levels specifying the different task interruption points. The resumption lag means differed significantly, $F(3$, $60)=9.38, p<.001$. The mean for the start-task interruption point was $1562 \mathrm{~ms}$, the mid-task mean was $1709 \mathrm{~ms}$, the end-task mean was $1737 \mathrm{~ms}$, and the scroll mean was $1475 \mathrm{~ms}$ (see Figure 3). Upon closer 
inspection of the classification system and the data, we felt that concluding a task was essentially the final portion of the mid-task performance. Therefore, we chose to combine these two levels for further analyses.

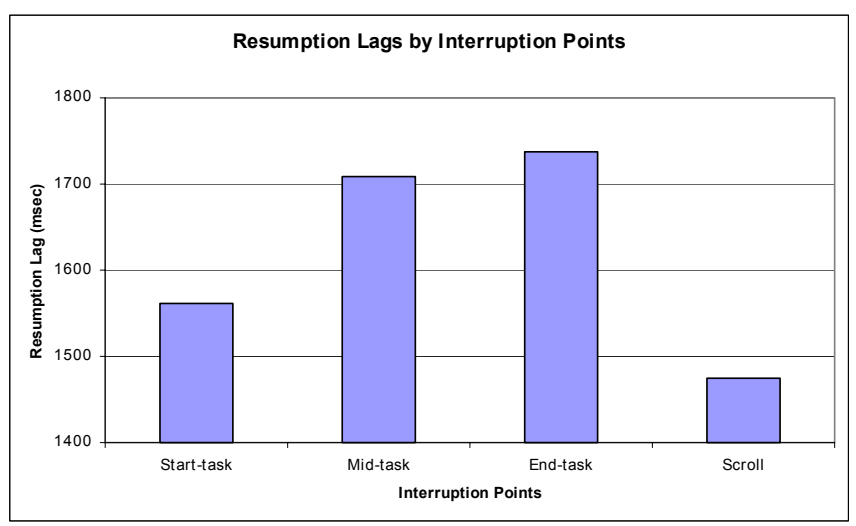

Figure 3. Resumption Lags by Interruption Points

The pairwise comparisons between the start-task, the newly formed mid/end-task, and the scroll interruption points using the Bonferroni adjustment revealed that the resumption lags associated with beginning a new task $\left(\mathrm{M}_{\text {Start }}=1562\right)$ were significantly less than the lags for the mid/end-task $\left(\mathrm{M}_{\mathrm{Mid} / \mathrm{End}}=1721\right)$, $p<.05$. The scroll lags $\left(\mathrm{M}_{\mathrm{Mid}}=1475\right)$ were also significantly less than the mid/end-task lags $\left(\mathrm{M}_{\mathrm{Mid} / \mathrm{End}}=1721\right), p<.05$. The start-task and scroll lags were not significantly different. These results demonstrate that the attention switching cost was lowest when interrupting VCR task performance at the beginning of a new task or when performing a repetitive operation within a task like scrolling through a list.

For the analysis of the total task time measure, only the tracking task difficulty factor was available because the interruption points factor was embedded within each trial. Each participant's trial time was recorded and the sum of the tracking task intervals was subtracted from the trial time to generate the total task time. Total task time is distinguished from the time-on-task shown in Figure 2 in that the resumption lags are included in the total task time. Although there were no differences between the easy and hard levels of the tracking task for resumption lags, the main effect for the tracking task difficulty for the total task time measure was significant, $F(1,20)=6.20, p<.05$. The mean task time for the hard tracking condition was 67.7 seconds, and 63.2 seconds for the easy condition. This result in combination with the lack of a tracking task difficulty main effect for the resumption lags suggests that there is indeed some interference from the loading task on the VCR task, but its effects are on the time-on-task intervals rather than the resumption lags.

\section{DISCUSSION}

The results clearly show that the point at which the VCR task is interrupted does matter in terms of the resulting resumption lags. It is better to interrupt a task before a new task is begun, or during a repetitive task like scrolling through a list. On the other hand, interrupting a task during the middle of a task or when a task is nearly completed will result in longer resumption lags. Because the VCR task maps to IVIS-type tasks reasonably well, these results give some hints as to the interruptability of specific IVIS-style task. However, in the context of drivers timesharing with an IVIS task, the drivers themselves control the interruption points. By understanding the potential costs of when the interruptions occur, designers can attempt to develop interfaces that have natural breaking points, such as short, discrete subtasks.

The fact that the task interruption points influenced the resumption lags shows that these lags can potentially be minimized by improved interface design. Research on task switching has shown that although switch costs can be reduced with visual and other cues, a bottom-up residual switch cost persists (Meiran, Chorev, \& Sapir, 2000). This suggests that the resumption lags may never be eliminated entirely. Additional research is required to determine the flexible range of resumption lags when switching between IVIS-style tasks and a driving task.

Even though the resumption lag measure may seem irrelevant when considering that it is simply a portion of the eyes-off-road time in the IVIS context, this study shows that resumption lags are flexible - longer or shorter depending on where in the task the interruptions occur. Longer resumption lags will lead to less time-ontask for each task exposure, or shutter open time, which in turn will lead to more shutter open intervals per task performance. In general, more shutter open intervals result in more eyes-off-road time. Whereas the TSOT measure is useful in predicting the eyes-off-road time for an IVIS task, the resumption lag measure can provide designers with additional sensitivity to one potential source of total task time variability.

Because there was no difference between the tracking task settings on resumption lag, the significant total task time difference provides an interesting clue to how the loading task may be affecting a user's ability to timeshare the tracking and VCR tasks. It can be inferred that the tracking task difficulty affected the time-on-task (shutter open time minus resumption lag - see Figure 2) variable because the hard tracking task resulted in significantly longer total task times, but not longer resumption lags. In the context of the visual occlusion technique, the TSOT measure would also be affected by 
the loading task difficulty even if the resumption lags were not. Whereas this study sheds some light on the issue, more research is required to clarify the effects of the loading task and interruption points on the time-ontask measure.

The establishment of the resumption lag as a factor in predicting an IVIS-style task time is also relevant to the GOMS modeling approaches pursued by Green (1999) and Nowakowski and Green (2001). This lag time can be incorporated into Green's model as the "mental" times used in GOMS modeling to yield more accurate predictions of total task time in dynamic settings.

The overall average resumption lag in this experiment was around 1600 milliseconds, which is a substantial amount of time when it has been shown that drivers often attempt to interlace IVIS tasks and driving in a series of one-second bursts (Gellatly \& Kleiss, 2000). Additional research is required before these findings with a VCR task can be generalized to IVIS tasks in the driving environment, but they do suggest that a switch cost may be present when IVIS-style tasks are interrupted at different stages.

\section{ACKNOWLEDGEMENTS}

This work was supported in part by grant number N0001400WX21058 to Greg Trafton from the Office of Naval Research. The views and conclusions contained in this document are those of the authors and should not be interpreted as necessarily representing the official policies, either expressed or implied, of the U. S. Navy.

\section{REFERENCES}

Altmann, E.M., and Trafton, J.G. (2002). Memory for goals: An activation-based model. Cognitive Science, 26, 39-83.

Green, P. and Tsimhoni, O. (2001). Visual occlusion to assess the demands of driving and tasks: the literature. Presentation at the Exploring the Occlusion Technique: Progress in Recent Research and Applications Workshop, Torino, Italy.

Available: http://www.umich.edu/ driving/occlusionworkshop2001/

Green, P. (1999). Estimating Compliance with the 15Second Rule for Driver-Interface Usability and Safety. Proceedings of the Human Factors and Ergonomics Society 43rd Annual Meeting. Santa Monica, CA: Human Factors and Ergonomics Society, CD-ROM.

Gellatly, A., and Kleiss, J. (2000). Visual attention demand evaluation of conventional and multifunction in-vehicle information systems. Proceedings of the IEA 2000/HFES 2000 Congress, 3, 282-285, Santa Monica: CA.

Karlsson, R., and Fichtenberg, N. (2001). How different occlusion intervals affect total shutter open time. Reg. no. 202-0006. Volvo Car Corporation. Presentation at the Exploring the Occlusion Technique: Progress in Recent Research and Applications Workshop, Torino, Italy. Available: http://www.umich.edu/ driving/occlusionworkshop2001/

Meiran, N., Chorev, Z., \& Sapir, A. (2000). Component processes in task switching. Cognitive Psychology, 41, 211-253.

Nowakowski, C. and Green, P. (2001). Prediction of Menu Selection Times Parked and While Driving Using the SAE J2365 Method (Technical Report 2000-49). Ann Arbor, MI, The University of Michigan Transportation Research Institute.

Wierwille, W. (1993). Visual and manual demands of the in-car controls and displays. In B. Peacock and W. Karwoski (Eds.), Automotive Ergonomics (pp. 299-320). Bristol, PA: Taylor \& Francis. 